\title{
RESEARCH ARTICLE \\ Nonlinear Model of Coastal Flooding by a Highly Turbulent Tsunami
}

\author{
Sergey A. Arsen'yev' ${ }^{1}$ Lev V. Eppelbaum²,
}

Received: 27 February 2021 / Accepted: 5 July 2021 / Published online: 10 August 2021

(c) The Author(s) 2021

\begin{abstract}
When a tsunami wave comes from ocean and propagates through the shelf, it is very important to predict several dangerous factors: (a) maximum flooding of the coast, (b) tsunami wave height on the coast, (c) velocity of the tsunami front propagation through the coast, and (d) time of tsunami arriving at a given point in the coast and around it. In this study we study the separate case where the angle of inclination $\alpha$ of the seacoast is equal to zero. A linear solution of this problem is unsatisfactory since it gives an infinite rate of the coastal inundation that means the coast is flooded instantly and without a frontal boundary. In this study, we propose a principally new exact analytical solution of this problem based on nonlinear theory for the reliable recognizing these essential tsunami characteristics. The obtained formulas indicate that the tsunami wave can be stopped (or very strongly eliminated) in the shelf zone until approaching the shoreline. For this aim, it is necessary to artificially raising several dozens of bottom protrusions to the level of the calm water.
\end{abstract}

Keywords Nonlinear long waves $\cdot$ Tsunami $\cdot$ Coastal inundation

Mathematics Subject Classification 35K55 $\cdot 86 \mathrm{~A} 05 \cdot 86 \mathrm{~A} 15 \cdot 76 \mathrm{~F} 40 \cdot 76 \mathrm{~B} 15$

Lev V. Eppelbaum

levap@tauex.tau.ac.il

Sergey A. Arsen'yev

Arsy7@mail.ru

1 Institute of the Physics of the Earth, Russian Academy of Sciences, Bolshaya Gruzinskaya St., Moscow 123995, Russia

2 Department of Geophysics, Faculty of Exact Sciences, Tel Aviv University, Ramat Aviv, 6997801 Tel Aviv, Israel

3 Azerbaijan State Oil and Industry University, 20 Azadlig Ave., Baku AZ1010, Azerbaijan 


\section{Introduction}

Tsunamis are the long gravitational waves in the ocean occurring because of a short-term change in its volume that is due to large-scale disturbances in the ocean surface its shores or the bottom $[8,31,39]$. Waves with a length $\lambda$ exceeding the depth of the ocean $H$ are called as long waves $(\lambda>H)$. Therefore, tsunami cover the entire ocean's thickness (in the concrete region) and can spread over transoceanic distances; therefore this is a planetary phenomenon like astronomical tides. Typical tsunami wave periods are from 1 min to several hours, and the characteristic wavelengths are from 1 to $100 \mathrm{~km}$. Therefore, when approaching the shelf, tsunami waves can nonlinearly interact with the shallow components of the ocean tide, which can weaken or strengthen the tsunami wave [5].

The tsunami phenomenon is a natural disaster that has been intensively studying since the second half of the twentieth century. The modern tsunami studies can be tentatively divided into three main groups.

First, tsunami sources in the oceans and seas are being studied (e.g., [2, 10, $30,47])$. Here, the waves are often calculated using the linear theory of potential motions of an ideal frictionless fluid under the gravity field influence $[6,31]$. Such models are called non-hydrostatic, since they do not use the shallow water equations and the hydrostatic law, which are valid for the long waves [3, 41]. In other models, the tsunami waves are considered as long waves already at the source of excitement. Therefore, they are called as the hydrostatic models [19, 32].

The second group of investigators studies the propagation of tsunami waves in ocean (e.g., [2, 10, 30, 31, 45]). Here the waves are considered as long ones, the hydrostatic models of the theory of shallow water are used, and the process itself substantially depends on the ocean's depth [37]. Both in the first and second group of the studies, the equations have a hyperbolic type. The friction on the ocean (marine) bottom is insignificant and is usually did not take into account.

In the third group of publications studies the processes of tsunami propagation through the continental shelf and coastal shallows, including the phenomena of transformation and destruction of the waves as well as the inundation of the coast [3, $5,13-15,28,33,35,42]$.

Our work belongs to the third group (the most dangerous to humanity) of the aforementioned studies. They are the most complex since they are usually based on solving nonlinear equations and contain a moving border-the water's edge line. On the other hand, the landfall stage of a tsunami is the most destructive, and its study is the most important from both a scientific and practical point of view. The tsunami approaches the shore zones in different forms depending on the structure of the coastal part of the shelf. If the shelf zone has a large depth up to the shore, then the tsunami simply floods the shore and calmly moving inland. At shallower depths of the shelf, the tsunami forms a solitary wave (soliton) in which the dispersion is balanced by the nonlinear accelerations $[6,8,20,34]$. The soliton moves cross the shelf, but when it approaches the shore, the nonlinear accelerations began to dominate. As a result, the higher harmonics arise and strongly distort the solitary wave. 
The soliton becomes unstable and collapses. At the same time, generates turbulence, and friction on the bottom becomes significant one. The tsunami wave transforms into a turbulent current that floods the coastal zone. However, it is significant that this water flow at this moment is no longer described by the hyperbolic equations. In this paper, we obtained a nonlinear parabolic equation describing the corresponding turbulent current, and got its exact solution.

Note that the process of the shore flooding is essentially depends on its geometry. For example, if the shore is a sheer cliff, then the height of the rising water will simply be equal to the doubled amplitude of the falling wave. If the height of the shore increases linearly towards the land, then the solution depends on the angle $\alpha$ of the shore inclination. In the numerous publications, as a rule is considered the case when $\alpha \neq 0$ [e.g., 10, 13, 15, 28, 31, 33, 37]. In this paper, we will look at the previously unexplored problem of the tsunami wave inundation of the flat plain considering the coastal slope absent $(\alpha=0)$. In the natural conditions, the flat coastal plains exist, for example, on the coasts of the Caspian Sea, the Sea of Azov and the Mediterranean Sea). The area of flooding and the range of tsunami propagation into the land are in this case is maximal.

In the intermediate cases, in the range $\pi / 2 \geq \alpha>0$, a reflected wave occurs, and the case $\alpha=\pi / 2$ corresponds to the vertical wall. The reflected wave greatly complicates the process of solving the problem [31]. The real topography of the coast and its horizontal inhomogeneity can be taken into account numerically using the powerful computers. At the same time, we can take into account all the forces in the equations of hydrodynamics including the nonlinear accelerations, the bottom friction, and the bottom roughness. However, along with the numerical methods, we must also use analytical solutions to test the numerical algorithms. Analytical solutions are also necessary for the engineering estimates of the coastal flooding magnitudes. These solutions allow us to understand how works the complex phenomenon of the natural disaster and to suggest reliable methods of dealing with its consequences.

Fig. 1 The horizontal coordinate axes and designations

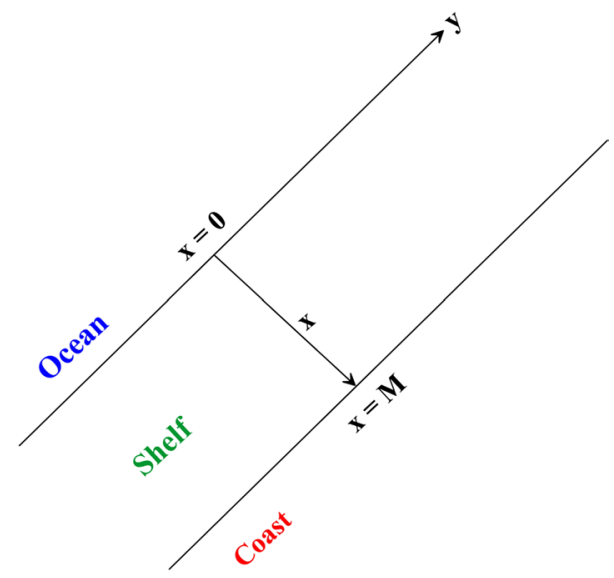




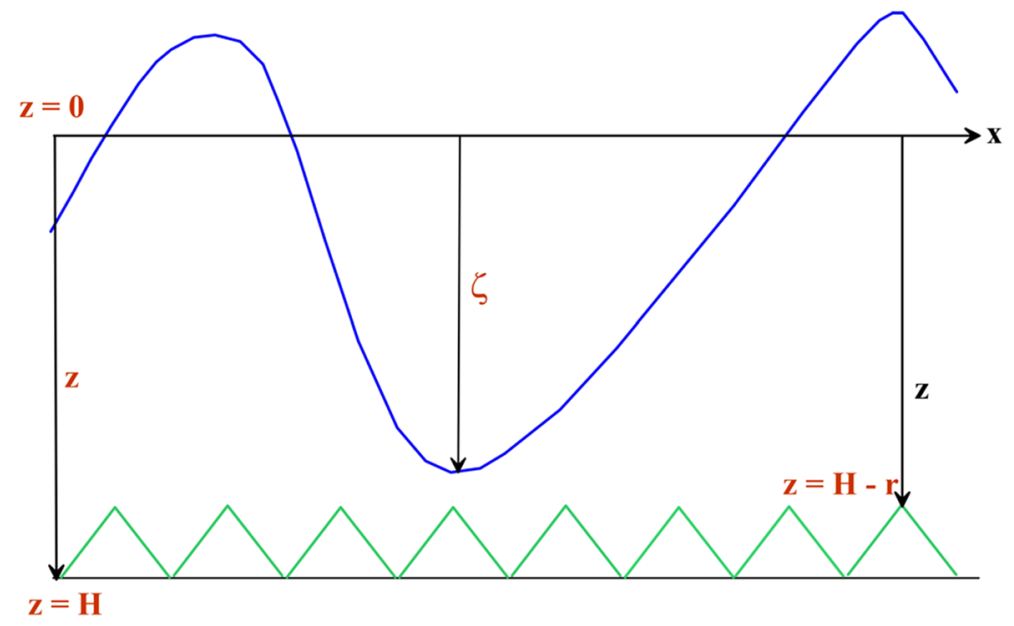

Fig. 2 Vertical section of the water flow and corresponding designations

\section{Statement of the Problem}

We choose the origin of coordinates at the sea edge of the shelf $x=0$. The $x$-axis is directed along the wave propagation direction perpendicular to the coast, the $y$-axis is perpendicular to the $x$-axis (left), the $z$-axis is down vertically (Figs. 1,2). The letter $M$ denotes the width of the shelf. Let us will select the level $z=0$ at the surface of the calm water, the letter $\zeta$ denotes the wave disturbance of the sea surface, and positive value $\zeta$ is counted down from the unperturbed level of $z=0$ (Fig. 2). The letter $H$ and $r$ denote the average depth of the shelf and the height of the proposed protrusions of the roughness at the bottom, respectively. Thus, the total depth of the shelf is value $H-r$.

We will use the equations of shallow water theory. They are obtained from equations of the geophysical hydrodynamics by integration along the $z$-axis in the range from $z=\zeta$ to $z=H-r[3,41]$. Assuming that there are no changes along the $y$-axis $\left(\frac{\partial}{\partial y}=0\right)$, we write the initial equations in the following form

$$
\begin{gathered}
\frac{\partial u}{\partial x}+\frac{\partial w}{\partial z}=0 \\
\frac{\partial u}{\partial t}+u \frac{\partial u}{\partial x}+w \frac{\partial u}{\partial z}=g \frac{\partial \varsigma}{\partial x}-\frac{1}{\rho} \frac{\partial p^{a}}{\partial x}-\frac{\partial R_{x}^{z}}{\partial z} .
\end{gathered}
$$

here $u$ is the component of the flow velocity in the wave along the $x$-axis, $w$ is the velocity component along the $z$-axis, $p^{a}$ is the atmospheric pressure at the water surface, $g$ is the gravity acceleration, $R_{x}^{z}$ is the vertical component of the turbulent Reynolds stresses [5], and $\rho$ is the density of water.

We introduce the characteristic scales of the time $t_{0}$ and pressure $p_{0}$, the scale of the longitudinal coordinate $L_{x}$, the scale of the vertical coordinate $H$, the scale of the horizontal current velocity $V_{\mathrm{x}}$, the scale of the vertical velocity of the water flow $V_{z}$, and 
the scale of the disturbance of the sea surface $\Lambda$. Next, we introduce the dimensionless variables

$$
T=t / t_{0}, X=x / L_{x}, Z=z / H, U=u / V_{x}, W=w / V_{z}, \Pi=p_{a} / p_{0}, \Lambda=\varsigma / H .
$$

Substituting equalities (2.3) into Eq. (2.1), we find

$$
\frac{V_{x}}{L_{x}}\left(\frac{\partial U}{\partial X}\right)+\frac{V_{z}}{H}\left(\frac{\partial W}{\partial Z}\right)=0 .
$$

Assuming that all terms of Eq. (2.4) have the same order, we obtain

$$
V_{z}=\left(\frac{H}{L_{x}}\right) V_{x}
$$

The vertical velocity is as many times less than the horizontal velocity, how many times the depth of the flow is less than its width.

Similarly, we substitute equalities (2.3) into Eq. (2.2), divide the result by the factor $V_{x}^{2} / L_{x}$ (the order of the nonlinear acceleration) and take into account formula (2.5). We get

$$
S h \frac{\partial U}{\partial T}+U \frac{\partial U}{\partial X}+\frac{1}{\mu} W \frac{\partial W}{\partial Z}=\frac{1}{F r} \frac{\partial \Lambda}{\partial X}+E u \frac{\partial \Pi}{\partial X}+\left(\frac{\mu}{\operatorname{Re}}\right) \frac{\partial^{2} U}{\partial Z^{2}} .
$$

here $S h=\frac{L_{x}}{V_{x} t_{0}}$ is the Strouhal number, $\mu=\frac{L_{x}}{H}$ is the geometrical parameter, $F r=\frac{V_{x}^{2}}{g H}$ is the Froude number, $\mathrm{Re}=V_{x} \frac{H}{A}$ is the Reynolds number of vertical turbulence, where $A$ is the coefficient of the vertical turbulent viscosity, and $E u=\frac{\rho_{0}}{\rho V_{x}^{2}}$ is the Euler number.

When deriving Eq. (2.6), we took into account that

$$
R_{z}^{x}=-A \frac{\partial u}{\partial z}
$$

We will accept the following values for typical scales: $t_{0}=1 \mathrm{~h}=3600 \mathrm{~s}$; $L_{x}=1 \mathrm{~km}=1000 \mathrm{~m} ; V_{x}=1 \mathrm{~m} / \mathrm{s} ; H=10 \mathrm{~m} ; p_{0}=10^{5} \mathrm{~Pa}$ (normal atmospheric pressure), $\rho=10^{3} \mathrm{~kg} / \mathrm{m}^{3}$. To estimate the coefficient of eddy viscosity $A$ in a strongly turbulent flow, we take the Felzenbaum's formula [6] $A=V_{x} H$. Therefore, $A=10$ $\mathrm{m}^{2} / \mathrm{s} ; S h=0.27 ; E u=100 ; \mu=100$ and $1 / \mu=0.01 ; 1 / F r=98 ; \operatorname{Re}=1 ; \mu / \operatorname{Re}=100$. As you can see, the left side of Eq. (2.6) is much smaller than its right. Therefore, in a strongly turbulent tsunami, Eq. (2.2) can be written as

$$
g \frac{\partial \varsigma}{\partial x}=\frac{\partial R_{x}^{z}}{\partial z}+\frac{1}{\rho} \frac{\partial p^{a}}{\partial x} .
$$

It is necessary to add the vertical boundary conditions to Eqs. (2.1) and (2.8):

$$
z=\varsigma, w=\frac{\partial \varsigma}{\partial t}+u \frac{\partial \varsigma}{\partial x}, R_{x}^{z}=R_{x}^{0}
$$




$$
z=H-r, u=w=0, R_{x}^{z}=R_{x}^{H} .
$$

Integrating Eqs. (2.1) and (2.8) along the vertical axis from $z=\zeta$ to $z=H-r$ (it is the real depth, taking into account the protrusions at the ocean bottom), we obtain

$$
\frac{\partial \varsigma}{\partial t}=\frac{\partial S}{\partial x} ; g(H-r-\varsigma) \frac{\partial \varsigma}{\partial x}-\left(\frac{H-r-\zeta}{\rho}\right) \frac{\partial p^{a}}{\partial x}=R_{x}^{H}-R_{x}^{0} .
$$

where $R_{x}^{0}$ is the turbulent stress on the surface of the water caused by the action of the wind.

When integrating, we took into account the boundary conditions (2.9) and (2.10). Here we introduce the total flow $S$ on a vertical:

$$
S=\int_{\varsigma}^{H-r} u d z
$$

Turbulent stresses on the sea surface $R_{x}^{0}$, as well as the atmospheric pressure gradient $\partial \rho^{a} / \partial x$ should be taken into account only when studying the processes of occurrence of the storm surges and meteorological tsunamis [7, 39]. In our case, when we study the tsunami wave approach to the shore, these terms can be neglected. We associate the water turbulent friction on the bottom $R_{x}^{H}$ with the total flow $S$ by a linear law $[7,21]$

$$
R_{x}^{H}=\omega_{T} S, \omega_{T}=\frac{3 A}{(H-r)^{2}} .
$$

This type of friction in the hydrodynamics is called as Rayleigh's friction, but Gill [21] notes that it was introduced even earlier by Airy [29] in the channel theory of the tides.

Thus, Eqs. (2.11) can be written as

$$
\frac{\partial \varsigma}{\partial t}=\frac{\partial S}{\partial x} ; \quad g(H-r-\varsigma) \frac{\partial \varsigma}{\partial x}=\omega_{T} S .
$$

Two Eqs. (2.14) can easily be reduced to one nonlinear equation of the parabolic type with respect to the level $\zeta$

$$
\frac{\partial \varsigma}{\partial t}=\frac{\partial}{\partial x}\left[K(\varsigma) \frac{\partial \varsigma}{\partial x}\right]
$$

in which the wave diffusion coefficient

$$
K(\varsigma)=\frac{g(H-r)}{\omega_{T}}-\left(\frac{g}{\omega_{T}}\right) \varsigma
$$

depends on an unknown quantity $\zeta$. 
The similar equations were studied in the static physics [11], in the theory of filtration $[9,12,38]$, in the theory of atomic explosions [43, 49], in the biomedical engineering and geophysics [26, 27] and in the theory of tornadoes [4]. To solve them, the numerical methods [43] and the approximate analytical methods $[9,38,49]$ have been developed. In this paper, we propose an elegant self-similar solution of the problem which describes the phenomenon under study with sufficient for the practice accuracy.

\section{Solution of the Problem}

We first consider the simple case of a deep shelf when $H-r>>\zeta$. Then Eq. (2.15) can be written as

$$
\frac{\partial \varsigma}{\partial t}=\kappa \frac{\partial^{2} \varsigma}{\partial x^{2}} .
$$

This is a classical parabolic equation of the type of the diffusion equation (or heat conduction) [16] which describes the process of tsunami wave dissipation on the shelf. It has the character of the turbulent spreading with a diffusion coefficient

$$
\kappa=\frac{g H(1-n)}{\omega_{T}}=\frac{g H^{3}(1-n)^{3}}{3 A} .
$$

Here $n=r / H$ is the relative roughness of the ocean bottom. This process can be understood by solving Eq. (3.1) with the corresponding initial (3.3) and boundary conditions (3.4):

$$
\begin{gathered}
\text { by } t \leq 0, \zeta=0 \text { for all } x, \\
\text { by } t>0: \zeta=\zeta_{0} \text { when } x=0 ; \zeta=0 \text { by } x \rightarrow \infty .
\end{gathered}
$$

As a result, we will be able to determine the coastal inundation having the maximum range of the tsunami propagation inland. We have [25, 43]

$$
\varsigma=\varsigma_{0}\left[1-\Phi\left(\frac{x}{2 \sqrt{\kappa t}}\right)\right],
$$

where

$$
\Phi\left(\frac{x}{2 \sqrt{\kappa t}}\right)=\frac{2}{\sqrt{\pi}} \int_{0}^{9} \exp \left(-\eta^{2}\right) d \eta
$$

is the probability integral in which the upper limit $\vartheta=\frac{x}{\left[2(\kappa t)^{1 / 2}\right]}$. 
The thickness of the coastal area flooded by the tsunami wave, i.e., the inundation zone $\delta$, can be found from the condition of a sufficiently noticeable decrease in the level $\zeta$ when moving away from the beginning $x=0$

$$
\varsigma=\varsigma_{0} \operatorname{erfc}\left(\frac{\delta}{2 \sqrt{\kappa t}}\right)=0.01 \varsigma_{0},
$$

where special function

$$
\operatorname{erfc}\left(\frac{x}{2 \sqrt{\kappa t}}\right)=1-\Phi\left(\frac{x}{2 \sqrt{\kappa t}}\right)
$$

is the additional probability integral.

The numerical value 0.01 is reached by the erfc function when the value of its $\operatorname{argument} \frac{x}{[4 \kappa t]^{1 / 2}}$ is equal to two. Hence,

$$
\delta=4 \sqrt{\kappa t}=4 \sqrt{\frac{g H(1-n) t}{\omega_{T}}},
$$

or

$$
\delta=4 \sqrt{\frac{g H^{3}(1-n)^{3} t}{3 A}} .
$$

It follows from Eq. (3.10) that the width of the flood zone $\delta$ does not depend on the amplitude of the tsunami wave $\zeta_{0}$ falling to the shelf zone, does not depend on the width of the shelf $M$, but very strongly depends on the depth of the shelf $H$, the relative roughness $n=\frac{r}{H}$ and the time $t$ of the tsunami action. Since tsunamis dissipate due to the turbulence, therefore with an increase in the shear turbulent viscosity coefficient $A$, the width of the flood zone $\delta$ decreases.

For $A=10 \mathrm{~m}^{2} / \mathrm{s}, n=0$ (smooth bottom) and $H=10 \mathrm{~m}$, from formula (3.10) follows that for $t=1 \mathrm{~h}, \delta=4300 \mathrm{~m}$. With a shelf width of $M=2000 \mathrm{~m}$, the coast will be flooded by $2300 \mathrm{~m}$. However, with a very rough bottom (reefs, rocky ledges at the bottom) when $n=0.5$, we have (for the same depth, time and turbulent viscosity) from formula (3.10) $\delta=1500 \mathrm{~m}$, i.e., a wave the tsunami completely attenuates on the shelf with a width of $M=2000 \mathrm{~m}$. We see that the tsunami attack can be stopped by creating the flood barriers or berms on the shelf with a height of $r=H$. In this case, $n=1,1-n=0$ and from formula (3.2) follows that $\kappa=0$.

Equation (3.6) gives

$$
\frac{2}{\sqrt{\pi}} \int_{0}^{\infty} \exp \left(-\eta^{2}\right) d \eta=1
$$

and from the solution of Eq. (3.5) we get $\zeta=0$. Thus, the tsunami inundation stops on the shelf, and the coast remains dry (intact). 
Note that the obtained solution is approximate and has two fundamental disadvantages. Firstly, the width of the flood zone, strictly speaking, is infinite one and we cut it off artificially using condition (3.7). Secondly, the water propagation through the shelf and shore has an infinite velocity, which is unrealistic one. These shortcomings belong to any solution to the degenerate linear parabolic Eq. (3.1). However, as we will see below, they are absent in the solution of the nonlinear Eq. (2.15).

Let us introduce the length scale $h=H-r$, time scale $T=h^{2} / A$, dimensionless coordinate $\varphi=x / h$ and dimensionless time $\tau=t / T$. Then the dimensionless diffusion coefficient

$$
\Delta=\frac{K}{A}=\frac{g h^{3}(1-e)}{3 A^{2}}=G L,
$$

where $e=\zeta / h$ is the dimensionless level disturbance, $G=\frac{g h^{3}}{3 A^{2}}$ is some parameter (the authors of the paper suggest to call it as 'Galileo's number'), and $L=1-e$ is the relative water surface level.

Then Eq. (2.15) takes the form

$$
\frac{\partial L}{\partial \tau}=G \frac{\partial}{\partial \varphi}\left(L \frac{\partial L}{\partial \varphi}\right) .
$$

Its solution

$$
\begin{gathered}
L(\varphi, \tau)=L_{0} \tau\left(1-\frac{\varphi}{c \tau}\right) \text { by } \varphi<c \tau, \\
L(\varphi, \tau)=0 \text { by } \varphi \geq c \tau .
\end{gathered}
$$

It is easy to verify that it satisfies not only Eq. (3.13), but the boundary conditions at the beginning of coordinates $x=0, \varphi=0$, and the initial condition $\tau=t=0$ :

$$
L(0, \tau)=L_{0} \tau, \quad L(\varphi, 0)=0,
$$

where $L_{0}$ is the initial constant value. For example, for $L_{0}=1$, we have $\zeta_{0}=0$, i.e., there is no any initial perturbation of the water surface level.

Indeed, substituting the solution (3.14) into Eq. (3.13), we obtain $c=\left(G L_{0}\right)^{1 / 2}$ and

$$
c=\frac{h}{A} \sqrt{\frac{g\left(h-\varsigma_{0}\right)}{3} .}
$$

The coordinates of the moving point $x^{*}$ of the water edge, that is the nose of the tsunami wave running onto the shore (where $L=1, \zeta=0$ ), is determined from the equation 


$$
1=L_{0} \tau\left(1-\frac{\varphi^{*}}{\tau}\right)
$$

which is equivalent to the following equation

$$
\varphi^{*}=c \tau-\frac{c}{L_{0}} .
$$

From this follows that $c=\frac{d \varphi^{*}}{d \tau}=\frac{T}{h} \frac{d x^{*}}{d t}$, or in the dimensional form

$$
V=\frac{d x^{*}}{d t}=\left(\frac{h}{T}\right) c=\sqrt{\frac{g\left(H-r-\varsigma_{0}\right)}{3}} .
$$

The tsunami nose coordinates (water edge) $x^{*}=\delta_{n}$ moves according to the law (3.19)

$$
x^{*}=\left(t-\frac{h^{2}}{A L_{0}}\right) \sqrt{\frac{g\left(H-r-\varsigma_{0}\right)}{3}},
$$

where $L_{0}=1-\left[\frac{\zeta_{0}}{H-r}\right]$.

\section{Discussions of the Results}

Solutions (3.14)-(3.21) describe simple, but actual physical-geodynamical model for the motion tsunami current on a coastal flatland with a finite velocity of (3.20). It differs from the Lagrange velocity $(g H)^{1 / 2}$ of the long waves, since here taken into account the roughness $r$, the initial perturbation of the water level $\zeta_{0}$ and the turbulent friction on the bottom. It can be seen from formula (3.17) that the tsunami wave with $\zeta_{0}=0$ can be eliminated by creating the roughness protrusions with a height of $r=H, h=0$ at the bottom of the shelf. However, in contrast to the linear case, the tsunami wave is not just scattered over the shelf, but stops because Eq. (3.20) indicates that its velocity $V$ vanishes. Here we should note that since we have neglected the several minor factors, the tsunami may not be stopped, but very much weakened (which practically means that these two events are of the equivalent importance).

Figure 3 shows the dependence of the level $D=-\zeta$ on the time $t$ for two distance $x: 300 \mathrm{~m}$ and $700 \mathrm{~m}$. The level $D$ is upward from the unperturbed sea surface in contrast to the level $\zeta$ which is down-directed (Fig. 2). Let us assume that we stand on the shore of a shelf with a width of $M=300 \mathrm{~m}$ near the water edge at the point $x=300 \mathrm{~m}$ from the beginning of coordinates $x=0$, which is located on the sea edge of this shelf (Fig. 1). We can see in Fig. 3 (curve 1) that the wave approaches the coast $x=300 \mathrm{~m}$ at the time $t=52 \mathrm{~s}$ with a negative phase. The value of $D$ becomes negative one and the water moves away from the coast, exposing the bottom of the shelf. This feature of the tsunami approach to the coast is well known from numerous observations. For example, during the tsunami on the island of Sumatra on December 24, 2004, the water first moved away from the coast and exposed the 


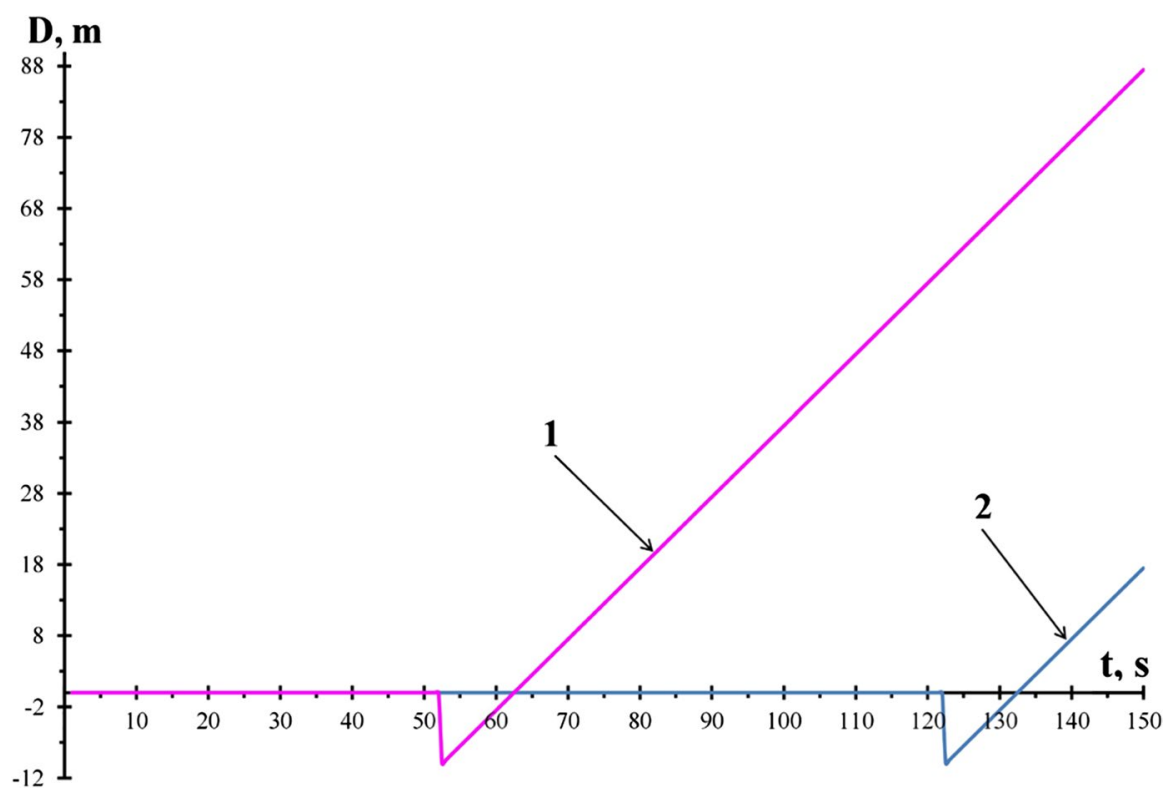

Fig. 3 Non-linear dependence of the sea level $D=-\zeta$ on the time $t$ at various distances from the origin $x=0$. Graph $1-\mathrm{x}=300 \mathrm{~m}$, the water edge is located at $t=52 \mathrm{~s}$. Graph $2-\mathrm{x}=700 \mathrm{~m}$, the water edge is located at $t=122 \mathrm{~s}$

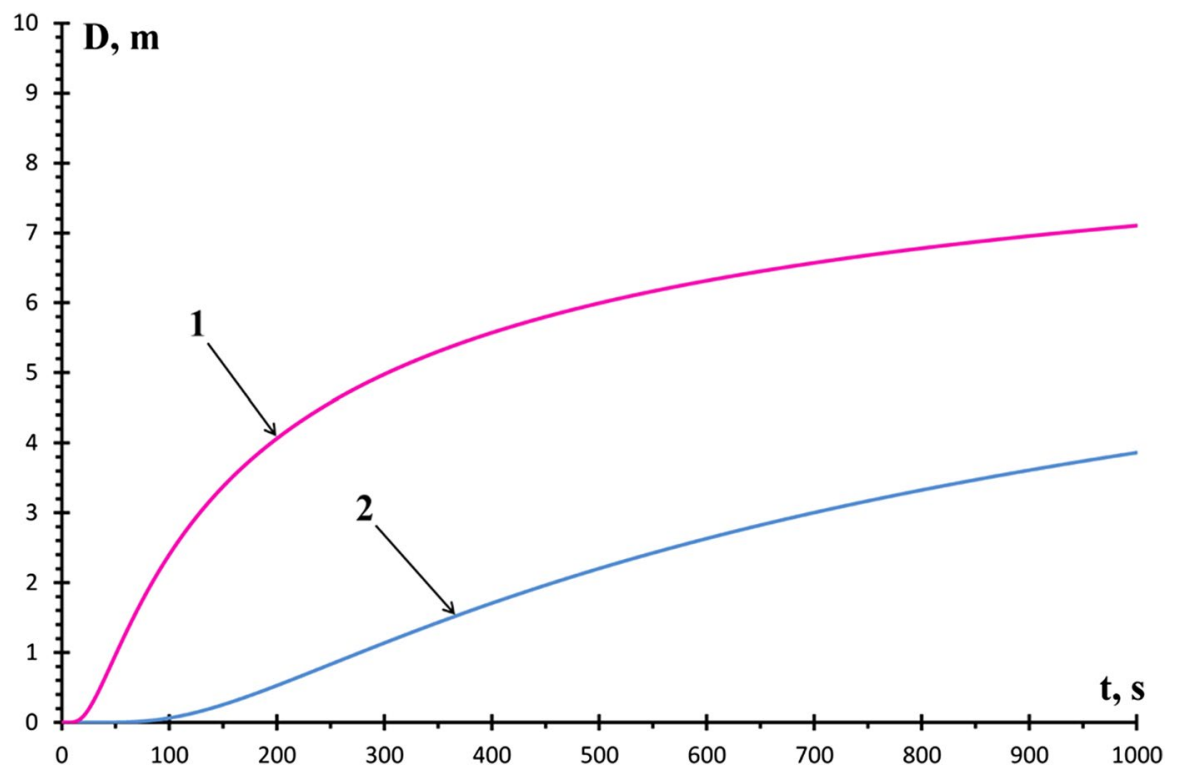

Fig. 4 Linear dependence of the sea level $D=-\zeta$ on the time $t$ at various distances from the origin $x=0$. Graph $1-x=300 \mathrm{~m}$. Graph $2-x=700 \mathrm{~m}$. Flooding of the coast occurs smoothly, there is no a tsunami front 
bottom of the shelf. And only after some time (10 s in Fig. 3, curve 1) did it attack the coast in the form of a rapidly growing, seething front. The linear solution of this phenomenon (Fig. 4) does not have this important feature and the coastal flooding occurs smoothly and stretched out in time.

At the moment of time $t=122 \mathrm{~s}$, the wave will reach the point $x=700 \mathrm{~m}$ from the origin of coordinates $x=0$ (Fig. 3, curve 2). The coastal flooding begins at the time $t=133 \mathrm{~s}$, and the wave will already be weakened due to the turbulent friction. If the water inflow stops at the origin of coordinates $L(x=0, t>80 \mathrm{~s})=1$, then the maximum flooding on the shore $(x=300 \mathrm{~m}$, Fig. 3, curve 1$)$ will reach a height of $20 \mathrm{~m}$. The wave will not reach the point $\mathrm{x}=700 \mathrm{~m}$ (Fig. 3, curve 2), and the coast will remain dry here.

The calculations shown in Fig. 3 are done for the case of $L_{0}=1, \zeta_{0}=0$, depth $h=10 \mathrm{~m}$ and velocity $V=5.71 \mathrm{~m} / \mathrm{s}$. Analyzing Fig. 3, we see that the region covered by the tsunami $\delta_{n}=x^{*}$ is finite and moves with a velocity $V$ described in Eq. (3.20). Let us compare the size of the flood zone $\delta$ according to the linear $\delta$ theory and the nonlinear theory $\delta_{n}$, setting $H=10 \mathrm{~m}, \theta_{0}=1, \zeta_{0}=0$ and $r=0$ (smooth bottom). According to the aforementioned nonlinear (more exact) theory, $V=5.71 \mathrm{~m} / \mathrm{s}$, and for $1 \mathrm{~h}$ tsunami will flood an area of the size $\delta_{\mathrm{n}}=20,529 \mathrm{~m}$. The linear theory presented in Eq. (3.10) gives for $A=10 \mathrm{~m}^{2} / \mathrm{s}$, the size of $\delta=4,300 \mathrm{~m}$, that is 4.7 times smaller (it is an erroneous solution).

For the very rough bottom, when $H=10 \mathrm{~m}, r=5 \mathrm{~m}, h=5 \mathrm{~m}, n=0.5$, we have $V=4 \mathrm{~m} / \mathrm{s}$ and the nonlinear theory gives $\delta_{\mathrm{n}}=14,500 \mathrm{~m}$. The linear theory at $A=10$ $\mathrm{m}^{2} / \mathrm{s}$ gives in this case $\delta=1,530 \mathrm{~m}$, that is 9.4 times less. As you can see, the linear approximation gives the great errors. The fact is that the diffusion coefficient $K$ in Eq. (2.15) stands near the highest derivative. Therefore, the solutions of this equation substantially depend on the value of $K(\zeta)$.

The solution of Eq. (3.14) also makes it easy to reconstruct the dependence of the depth $D$ on the distance $x$ at the various fixed times t from the disturbing source. The corresponding nomograms and graphs can be employed for the engineering assessments for construction of the artificial structures that should protect the especially significant objects (for example, nuclear and thermal power plants, chemical plants, airfields and others targets $[8,42])$, located nearly the shores of the seas and oceans, from the dangerous tsunami phenomenon.

Comparing Figs. 3, 4, we see the significant differences between the nonlinear solution presented in (3.14) and (3.15) and the linear solution shown in (3.7). In Fig. 4, there is no water rollback at the leading edge of the wave which is observed in nature. The character of coastal flooding according to the linear solution (3.7) looks like a diffusion spreading of the sea surface level disturbance $\varsigma_{0}$ at the outer edge of the shelf $x=0$. The speed of propagation of the disturbance $\varsigma_{0}$ is infinitely large (long-range action). We must note that Eq. (3.1) is a parabolic equation, while waves are usually described by hyperbolic equations [48].

From the point of view of the general theory, the nonlinear equations presented in (2.15) and (2.16) belongs to the class of nonlinear equations describing the time evolution of dynamical systems of various nature [22, 23] 


$$
d \mathbf{Q} \frac{(\mathbf{X}, t)}{d t}=F(\mathbf{Q}, \nabla, \beta, \mathbf{X}, t) .
$$

Here $\mathbf{Q}$ is the state vector of the system, $\mathbf{X}$ is the radius-vector of a point in a space $(x, y, z), \beta$ are the some parameters characterizing the system. The operator $\nabla$ takes into account the spatial derivatives describing, for example, diffusion. In addition, the right-hand side of Eq. (3.22) is a nonlinear function of the state vector of the system Q. Depending on the type of this nonlinearity, various dynamic structures, called autowaves, can arise. These include, for example, excitation impulses in nerves, moving fronts of combustion and flames, spiral vortices, cells of various nature, plane and circular autowaves moving at a certain speed, rotating and pulsating autowaves. They can occur in continuous media, in biological systems (morphogenesis, work muscles), chemical reactors, in physics and in technical processes (combustion of various types, heterogeneous catalysis, plasma, information transfer, optoelectronic lines), in society (spread of rumors), in medicine (spread of epidemics, conduction system of the heart, neural network), geophysics and other sciences [e.g., 1, 16, 22-24, 26, 27, 36, 44, 46, 50].

It is easy to see that Eqs. (2.15) and (2.16) can be reduced to the standard form describing autowaves [50]

$$
\frac{\partial \zeta}{\partial t}=\frac{1}{R} f(\zeta)+\kappa \frac{\partial^{2} \zeta}{\partial x^{2}},
$$

where

$$
f(\zeta)=-\frac{g}{\omega_{T}^{2}}\left[\left(\frac{\partial \zeta}{\partial x}\right)^{2}+\zeta \frac{\partial^{2} \zeta}{\partial x^{2}}\right]=-\frac{g}{2 \omega_{T}} \frac{\partial^{2} \zeta^{2}}{\partial x^{2}}
$$

where $f(\zeta)$ the nonlinear function of sea level $\zeta$ and its derivatives, $R=1 / \omega_{T}$ is the relaxation time, and $\kappa$ is the diffusion coefficient [see Eq. (3.2)]. The duration of autowaves is of the order of $R$, and the speed of movement is of the order

$$
\left(\frac{k}{R}\right)^{1 / 2}=[g(H-r)]^{1 / 2}
$$

Lagrange in 1781 derived a similar formula $(g H)^{1 / 2}$ for the speed of long gravitational waves (tides, tsunamis) in the open ocean $[21,29]$. On a rough shallowwater shelf and on the coast, these waves become turbulent, and a more accurate formula (3.30) takes place for their velocity calculation. Thus, solution presented in Eqs. (3.14) and (3.15) describes a tsunami wave moving at a speed close to the speed of long gravitational waves in the open ocean. However, due to the collapse of the wave on the shallow, rough shelf, it turns into a turbulent current that floods the coast. In this case, the type of equation is changed. The hyperbolic equation typical for waves in the open ocean is replaced by a nonlinear parabolic equation describing the tsunami wave run up to the coast. 


\section{Conclusions}

Let us state the main results obtained in this paper. Based on the nonlinear theory of the shallow water and taking into account the strong turbulent friction on a rough bottom, the theory of tsunami roll-up to a flat shore $(\alpha=0)$ is extended. Exact analytical solutions for the linearized and nonlinear equations are found. It is shown that the use of solutions of the linearized equations leads to significant errors. The obtained formulas make it easy to calculate the advancement of the water front inland, the height of flooding of the shelf and shore at a given point, the tsunami wave propagation range, the average current velocity in the wave, and other characteristics necessary for the concrete engineering calculations. It was established that the velocity of the tsunami wave can be turned to zero, that is the movement of the tsunami wave can be stopped (or very much weakened) when approaching the ocean coast. To realize this, it is necessary to increase the height of the roughness protrusions (possibly using the artificial adjustable structures) on the bottom of the shelf $r$ to the level of undisturbed depth of the shelf $H$. Therefore, the strong turbulent friction will destroy the tsunami wave on the shelf and it does not reach the shore. We suggest that the obtained solution is essential for the development of artificial constructions in the potential tsunami-dangerous coastal zones.

Acknowledgements The authors would like to thank anonymous reviewers, who thoroughly reviewed the manuscript, and their critical comments and valuable suggestions were very helpful in preparing this paper. We acknowledge the Institute of the Physics of the Earth (Moscow) and the Faculty of Exact Sciences of Tel Aviv University for supporting this investigation.

\section{Declarations}

Conflict of interest The authors declare no conflicts of interest.

Open Access This article is licensed under a Creative Commons Attribution 4.0 International License, which permits use, sharing, adaptation, distribution and reproduction in any medium or format, as long as you give appropriate credit to the original author(s) and the source, provide a link to the Creative Commons licence, and indicate if changes were made. The images or other third party material in this article are included in the article's Creative Commons licence, unless indicated otherwise in a credit line to the material. If material is not included in the article's Creative Commons licence and your intended use is not permitted by statutory regulation or exceeds the permitted use, you will need to obtain permission directly from the copyright holder. To view a copy of this licence, visit http://creativecommons.org/licen ses/by/4.0/.

\section{References}

1. Achromeyeva, T.S., Kurdumov, S.P., Malinetsky, G.G., Samarsky, A.A.: Structures and Chaos in nonlinear media. FIZMATLIT, Moscow (2007) (in Russian)

2. Allgeyer, S., Cummins, P.: Numerical tsunami simulation including elastic lading and seas water density stratification. Geophys. Res. Lett. 41, 2308-2375 (2014)

3. Arsen'yev, S. A.: On the nonlinear equations of long sea waves, Water Resour (1): 29-35 (1991) (in Russian) 
4. Arsen'yev, S. A., Babkin, V. A., Gubar, A. Yu., Nikolaevskiy, V. N.: Theory of Mesoscale Turbulence. Eddies of the Atmosphere and the Ocean. In: Golitsyn, G.S. (Ed.). Regular and Chaotic Dynamics. Inst. of Computer Sciences, Moscow-Izhevsk, (2010) (in Russian)

5. Arsen'yev, S.A., Rykunov, L.N., Shelkovnikov, N.K.: Nonlinear interactions of tsunami and tides in an ocean. Doklady Russian Acad. Sci. 331(6), 732-734 (1993)

6. Arsen'yev, S.A., Shelkovnikov, N.K.: The second approximation in the theory of solitary waves on water. Moscow Univ. Phys. Bull. 44(1), 52-54 (2004)

7. Arsen'yev, S.A., Shelkovnikov, N.K.: Storm surges as dissipative solitons. Moscow Univ. Physics Bull. 68(6), 483-489 (2010)

8. Arsen'yev, S.A., Zhivogina, O.A., Seleverstov, S.V., Shelkovnikov, N.K.: Power influence of tsunami waves on the nuclear station. At. Energ. 85(1), 69-72 (1998) (in Russian)

9. Barenblatt, G.I.: Scaling, self-similarity, and intermediate asymptotic. Cambridge Univ. Press, Cambridge (1996)

10. Beisel, S., Chubarov, L., Didenkulova, I., Kil, E., Levin, A., Pelinovsky, E., Shokin, Y., Sladkevich, M.: The 1956 Greek tsunami recorded at Yafo, Israel, and its numerical modeling. J. Geophys Res 114(CO 9092), 1-18 (2009). https://doi.org/10.1029/2008JC005262

11. Boltzmann, L.: Lectures on gas theory. Dover Publ Inc. NY, USA (2011)

12. Boussinesq, J.: Recherches theoriques sur L'ecoulement des nappes d'ean infiltrees dans le sol, J. de Math. Pures et Appl., Ser. 5, t. X, fasc.1 (1904)

13. Carrier, G.F., Greenspan, H.P.: Water waves of finite amplitude on a sloping beach. J. Fluid Mech. 4, 97-109 (1958)

14. Choi, B.H., Hong, S.I., Pelinovsky, E.: Distribution of run up heights of the December 26, 2004 tsunami in the Indian Ocean. Geophys. Res. Lett. 33(L13601), 1-4 (2006). https://doi.org/10.1029/ 2006GL025867

15. Didenkulova, I., Pelinovsky, E.: Tsunami run-up on a plane beach in a tidal Environment. Pure Appl. Geophys. 177, 1583-1593 (2020). https://doi.org/10.1007/s00024-019-02332-y

16. Eppelbaum, L.V., Kutasov, I.M., Pilchin, A.N.: Applied geothermics. Springer, Heidelberg, New York (2014)

17. Felzenbaum, A.I.: Theoretical foundations and methods for calculating the steady-state SeaCurrents. USSR Academy of Sciences, Moscow (1960) (in Russian)

18. Fife, P.C.: Mathematical aspects of reacting and diffusion. Systems lecture notes in biomath, vol. 28. Springer, Berlin, Heidelberg, New York (1979)

19. Garagash, L. A., Lobkovsky, L. I.: An analysis of the dynamic displacement process of the sea bottom due to a subduction zone earthquake, Trans. of the 4th Int. FLAC Symposium on Numerical Modelling in Geomechanics (H. Varona, Ed.), Paper 06-01. Itaca Consulting Groups. Inc. Minneapolis, USA, (2006) 1-5

20. Geyer, A.: Solitary traveling water waves of moderate amplitude. J. Nonlinear Math. Phys. 19(Suppl. 1), 104-115 (2012)

21. Gill, A.E.: Atmosphere-ocean dynamics. Academic Press, New York, London, Paris (1982)

22. Haken, H.: Synergetics. Introduction and advanced topics. Part 1. Non equilibrium phase transitions and self-organization in physics, chemistry and biology. Springer, Berlin, Heidelberg, New York (1978)

23. Haken, H.: Synergetics. Introduction and advanced topics. Part II. Advanced topics instability hierarchies of self-organizing systems and devises. Springer, Berlin, Heidelberg, New York (2004)

24. Hirsch, M.W., Smale, S.: Differential equations, dynamical systems, and linear algebra. Academic Press, New York (1974)

25. Jeffreys, H., Swirles, B.: Methods of mathematical physics. Cambridge Univ. Press, Cambridge (1966)

26. Kardashov, V. R., Einav, S., Eppelbaum, L. V., Ismail-Zadeh, A.: Novel approach to investigation and control of nonlinear nonstationary processes: application to environments and biomedical engineering, Sci. Israel (3), 24-33 (1999)

27. Kardashov, V.R., Eppelbaum, L.V., Vasilyev, O.V.: The role of nonlinear source terms in geophysics. Geophys Res Lett 27(14), 2069-2072 (2000)

28. Korycansky, D.G., Lynett, P.J.: Run-up from impact tsunami. Geophys. J. Int. 170, 1076-1088 (2007)

29. Lamb, H.: Hydrodynamics, 6th edn. Cambridge Univ. Press, London (1932) 
30. Lay, T., Li, L., Cheung, K.F.: Modelling tsunami observations to evaluate a proposed late tsunami earthquake stage for the 16 September 2015 Illapel, Chile, Mw 8.3 earthquake. Geophys. Res. Lett. 43, 7902-7912 (2016)

31. Levin, B.W., Nosov, M.A.: Physics of tsunami. Springer, AG Switzerland (2016)

32. Lobkovsky, L.I., Garagash, I.A., Mazova, RKh.: Numerical simulation of tsunami waves generated by the underwater landslide for the Northern Coast of the Black Sea (Dzhubga Area). Geophys. J. Int. 218, 1298-1306 (2019)

33. Montoya, L., Lynett, P.: Tsunami versus infragravity surge: comparison of the physical character of extreme run up. Geophys. Res. Lett. 45(23), 12982-12990 (2018)

34. Nachbin, A., da Silva SimÕes, V.: Solitary waves in open channels with abrupt turns and branching points. J. Nonlinear Math. Phys. 19(Suppl. 1), 116-136 (2012)

35. Namekar, S., Yamasaki, Y., Cheung, K.C.: Neural network for tsunami and run up forecast. Geophys. Res. Lett. 36(L08604), 1-6 (2009)

36. Nicolis, G., Prigogine, I.: Self-organization of non-equilibrium systems. John Wiley \& Sons, London, Sydney, Toronto (1977)

37. Pelinovsky, E.N.: Hydrodynamics of Tsunami Waves. Inst. of Applied Physics of the Russ. Acad. of Sci., Nizhny Novgorod, Russia, (1996) (in Russian)

38. Polubarinova-Kochina, P.Y.: Theory of ground water movement. Nauka, Moscow (1971). (in Russian)

39. Rabinovich, A.B.: Twenty-seven years of progress in the science of meteorological tsunami following the 1992 Daytona Beach events. Pure Appl Geophys 177, 1193-1230 (2020)

40. Reynolds, O.: On the dynamical theory of incompressible viscous fluids and the determination of the criterion. Philosoph. Trans. Royal Soc. London 186, 123-161 (1894)

41. Røed, L.P.: Fundamentals of atmospheres and oceans on computers. Institute of Geosciences, University of Oslo, Norway, (2014)

42. Satake, K., Fujii, Y., Harada, T., Namegaya, Y.: Time and space distribution of coseismic slip of the 2011 Tohoku earthquake as inferred from tsunami waveform data. Bull. Seismol. Soc Am 103(2B), $1165-1170$ (2013)

43. Tikhonov, A.N., Samarsky, A.A.: Equations of mathematical physics. Pergamon Press, Oxford (1963)

44. Vasiliev, V.A., Romanovsky, Yu.M., Yakhno, V.G.: Autowave processes in distributed kinetic systems. Adv. Phys. Sci. 128(4), 625-666 (1979)

45. Wang, Y., Satake, K., Maeda, T., Gusman, A.B.: Green's function—based tsunami data assimilation: a fast data assimilation approach toward tsunami early warning. Geophys. Res. Lett. 44, 10282-10289 (2017)

46. Weidlich, W., Hang, G.: Quantitative Sociology. Springer Series in Synergetics, Vol. 14, Springer, (1983)

47. Wendt, J., Oglesby, D.D., Geist, E.L.: Tsunami and splay fault dynamics. Geophys. Res. Lett. 36(L15303), 1-5 (2009)

48. Whitham, G.B.: Linear and nonlinear waves. John Wiley \& Sons, London, Sydney, Toronto (1974)

49. Zeldovich, Ya. B., Companaetz, A. S.: On the theory of heat propagation with temperaturedepended thermal conductivity, Coll. of papers dedicated to the 70th anniversary of Academician A. F. Ioffe. USSR Acad. of Sci., Moscow (1950) 61-71 (in Russian)

50. Zhabotinsky, A.M.: Autowaves. In: Prokhorov, A.M. (ed.) Physical encyclopedia, vol. 1, pp. 11-12. The Soviet Encyclopedia, Moscow (1988) (in Russian) 